\title{
DINÁMICA POBLACIONAL, ESTRUCTURA AGRARIA Y DESARROLLO AGRÍCOLA EN BRASIL
}

\author{
Vinicius CALDEIRA BRANT \\ CEBRAP
}

UNA DIFICULTAD inicial en la discusión de la literatura brasileña sobre las relaciones entre la dinámica poblacional y la estructura agraria, surge del tratamiento vago e indirecto del tema en la mayor parte de los estudios disponibles. ${ }^{1}$ La investigación sobre las transformaciones en la agricultura y la discusión sobre las características de la estructura agraria en el Brasil han ocupado parte importante del trabajo en diversas ramas de la ciencia social. Otras investigaciones demográficas y estudios económicos, sociológicos y antropológicos sobre temas poblacionales, en especial los referentes a las migraciones, a las relaciones ciudad-campo y a la formación de fuerza de trabajo, se encuentran ante la necesidad de definir el cuadro rural y sus implicaciones. Sin embargo, la literatura sobre dinámica poblacional y desarrollo agrícola está, por regla, dicotomizada. Y, a menos que el analista se disponga a una reordenación de los datos parciales de información presentes en estudios enfocados en diversas direcciones - lo que constituiría por sí una nueva investigación - tendrá que contentarse con una selección limitada de trabajos específicos.

Por otra parte, es preciso considerar que la propia ordenación de la información disponible implica algunos problemas. Las dificultades teóricas suponen la imposibilidad de reducción metodológica de perspectivas a veces no sólo diversificadas, sino opuestas en ocasiones en cuanto a los

1 El examen de los trabajos comentados se hizo a partir del inventario de las investigaciones sobre problemas poblacionales relevantes para políticas de población, realizado en fecha reciente para pispal. El trabajo realizado en Brasil incluyó el examen de cerca de 1500 estudios concluidos en el período 1960-1974. De éstos, un reducido número interesa a la temática de este Seminario: apenas algunas decenas tratan de manera indirecta y aproximada sobre las relaciones entre dinámica poblacional y estructura agraria $\mathrm{y}$, un número muy reducido trata directamente sobre estas relaciones. Los trabajos citados son examinados a título de ejemplo, sin la preocupación de establecer una lista sistemática o exahustiva. Datos más concretos se pueden encontrar en el trabajo próximo a publicarse por PISPAL: "Levantamiento de estudios terminados y en curso sobre problemas de población relevantes para políticas de población en América Latina”. 
marcos de análisis. Aparecen dificultades prácticas desde el primer examen de la calidad de los datos primarios, en relación a varios aspectos relevantes. La especulación no puede sustituir a la investigación y, por lo tanto, un balance del conocimiento sobre las relaciones entre las variables demográficas y el desarrollo agrícola en el Brasil se vuelve más una exposición de lagunas e interrogantes que propiamente una sistematización de la información.

Desde el punto de vista de los datos demográficos básicos, la mayor parte de los conocimientos sobre el campo brasileño es residual. Con excepción de los cinco censos agrícolas realizados a partir de $1920,{ }^{2}$ son escasas las informaciones directas sobre la población rural. La mayoría de los estudios sobre esa población y sus índices de crecimiento están hechos a través de cálculos con base en los datos sobre la población en general, descontada la población urbana.

La escasez de informaciones hace que la mayor parte de los análisis, basados en cálculos y proyecciones de la población rural, se sitúen en un terreno en extremo genérico, de comentarios sobre la disminución relativa de la población rural, a causa del proceso de urbanización. A su vez, cuando se examinan los estudios sobre las variables demográficas básicas que interfieren en ese crecimiento, encontramos algo más que la desagregación de los datos censados, a través de artificios metodológicos destinados a suplir la carencia de información directa, además de levantamientos sobre las poblaciones urbanas que eventualmente incluyen, en el examen diferencial del universo en estudio, datos sobre la parte de origen rural.

Son raros los trabajos que examinan de manera específica la fecundidad en las áreas rurales y, desde el punto de vista explicativo, casi siempre se limitan a formular hipótesis genéricas sobre los factores que interfieren en la variación de las tasas. ${ }^{3}$ En algunos estudios sobre la

2 En $1920,1940,1950,1960$ y 1970.

3 La exploración de los datos censales se ha hecho de manera sistemática por el IBGE. Véanse por ejemplo, los estudios sobre fecundidad urbana y rural, en varios Estados, publicados en Brasil, CNE, Laboratorio de estadística, Pesquisas sobre a natalidade no Brasil, 4: serie, Río de Janeiro, IBGE, 1965; Giorgio Mortara, "A fecundidade da mulher, nas populaçōes urbanas e rurais do Brasil, segundo as unidades da Federação", Río de Janeiro, IBGE, 1962, Estudos demográficos, 270 (Mimeo). Un estudio específico con base en datos censales para los Estados de la frontera agrícola busca relacionar las elevadas tasas de fecundidad en esas regiones con la disponibilidad de tierras y mejores condiciones económicas de las familias de inmigrantes: Thomas William Merrick, "Fertility Petterns on the Brazilian Agricultural Frontier, 1950 to 1970", Belo Horizonte, cEDEPLAR (Mimeo), 1973. Una tentativa por estudiar las variaciones en la fecundidad en un continuum rural-urbanoindustrializado en relación con variables estructurales y socio-psicológicas, fue hecho a través de una encuesta con 726 mujeres, no solteras y fecundas, en cinco comunidades brasileñas de diferentes tamaños, incluida una villa rural: Bernard C. Rosen, et al., "Industrialization, Family and Fertility: A Structural-Psychological Analysis of the Brazilian Case", Demography, 8, No 1, febrero de 1971, pp. 49-69. 
fecundidad urbana, aparecen referencias indirectas sobre los patrones de fecundidad en los lugares de origen de los emigrantes venidos de las áreas rurales. Esas referencias surgen de la tentativa de explicar las diferencias de fecundidad entre emigrantes y no emigrantes, encontradas en levantamientos de muestras en las ciudades. Mas, como las relaciones establecidas entre las variaciones de la fecundidad y el estatus migratorio son casi siempre poco significativas, la atención dedicada de manera circunstancial a la fecundidad en las áreas rurales es muy superficial en este caso. ${ }^{4}$ Casi siempre la explicación insiste en la influencia de los patrones culturales "tradicionales", vigentes en el campo, en la diferenciación de los niveles de fecundidad rurales y urbanos.

En lo que se refiere a la mortalidad, el conocimiento sobre las áreas rurales es genérico e indirecto. Sólo se dispone de estadísticas directas de las defunciones para los municipios de las capitales y algunas grandes ciudades. Los pocos levantamientos específicos se limitan a algunas áreas urbanas.

Es en el estudio de las migraciones, en que la atención directa sobre las condiciones de las áreas rurales se manifiesta con mayor frecuencia. Se trata de una atención obligatoria, tanto en el caso de las migraciones internacionales, como en el de las migraciones internas. La localización de los inmigrantes extranjeros en áreas laborales y en colonias agrícolas, constituyó casi siempre el objetivo de las políticas migratorias gubernamentales, y a pesar de importantes flujos de esos inmigrantes en dirección a las ciudades, aún hoy se encuentran muchos núcleos concentrados, en especial en las áreas de colonización. En lo que se refiere a las migraciones internas, el interés en relación a las áreas rurales es aún más obvio: el campo ha sido de manera permanente la principal área de origen de esas migraciones. Durante varios decenios las migraciones rurales ur-

4 Existen algunas excepciones: en estudio especifico sobre la fecundidad diferencial, según el origen urbano o rural, con base en datos de la investigación sobre" "Reprodução humana no Distrito de Sāo Paulo" (1965-1966), concluye que la fecundidad de las mujeres de São Paulo se diferencia según su origen, pero no está influida por las pautas de fecundidad de la localidad de origen. La atención se dirige así, de preferencia, hacia las normas de la transición en el comportamiento en el medio urbano. Véase: Cecilia Helena Ornellas Renner, Migração ruralurbana e fertilidade em São Paulo, São Paulo, 1972, Facultad de Salud Pública de la Universidad de São Paulo, tesis doctoral, inédita. Una encuesta efectuada en un barrio de la ciudad de Campinas, estudia de manera específica las variaciones de la fecundidad en relación con el origen urbano o rural de los cónyuges. Véase Reginaldo Zaccara de Campos, Fatores sociais $e$ variações na fecundidade e no tamanho da família, Campinas, Facultad de Ciencias Médicas de la Universidad Estatal de Campinas, 1971, tesis doctoral, inédita. Otro estudio sobre relaciones entre fecundidad urbana y origen rural fue realizado en seis ciudades brasileñas. Se estudia edad, factores de estatus y tamaño de la ciudad, en relación con origen urbano y rural y variaciones en la fecundidad: Sugiyama Iutaka, et al., "Factors Affecting Fertility of Natives and Migrants in Urban Brazil", Population Studies, 25, № 1 , marzo de 1971, pp. 55-62. 
banas constituyeron la principal corriente migratoria. Por otro lado, la intensificación reciente de los flujos poblacionales que se dirigen a las áreas de frontera agrícola, acentúa el interés por las migraciones rurales. Con todo, también aquí las lagunas e interrogantes son importantes.

Aunque haya un número razonable de estudios sobre las migraciones internacionales que centran la atención en las áreas agrícolas de destino, tienden a concentrar mayor interés en los grupos de origen. ${ }^{5}$ La concentración espacial de los emigrantes en esas áreas, por sí misma, rara vez es objeto de estudio, lo que se explica en parte por tratarse del resultado obvio de procesos dirigidos (política migratoria gubernamental o importación privada de mano de obra). Pero, dado el enfoque con frecuencia dirigido a las características individuales de los emigrantes, a veces da la impresión de"que en ciertos estudios específicos se busca más la economía de tiempo y de recursos en la realización de las entrevistas que el análisis de la organización productiva o social del área. Por otro lado, la preocupación de realzar los rasgos específicos de los emigrantes, lleva

5 Entre los estudios sobre las migraciones internacionales de interés para el conocimiento de la agricultura en los lugares de destino, se pueden citar varios trabajos presentados en el $4^{\circ}$ Simposio Nacional de Profesores Universitarios de Historia, realizado en 1968 en Porto Alegre: Colonização e migração, São Paulo, USP, 1969, en especial Eduardo d'Oliveira, França, "Engenhos, colonização e cristãos-novos na Bahia Colonial" (pp. 181-241) en donde se analiza la influencia del espíritu burgués de los cristianos, nuevos en la economía mercantil de los ingenios, en la región del Recôncavo; Dante de Laytano, "Colonização Açoriana no Rio Grande do Sul” (pp. 391-421) que discute la influencia açoriana en ciertos cultivos y en la ganadería; Jean Roche, "Colonização e migração no Rio Grande do Sul" (pp. 745-756), en donde se estudia la agricultura basada en la pequeña propiedad a partir' de la organización social de los colonos alemanes; Oksana Boruszenko, "A imigração ucraniana no Paraná" (pp. 423-439), trabajo donde se resalta el contraste entre el estancamiento económico en las áreas tradicionales de colonización y la prosperidad de los emigrantes que se dirigirán posteriormente a la nueva frontera agrícola. En varios estudios sobre los inmigrantes japoneses se analiza la organización de la producción agrícola y la influencia económica y cultural de los colonos de esa nacionalidad en los lugares de destino. Véase en particular, Hiroshi Saito, $O$ Japonês no Brasil; estudo de mobilidades e fixação, São Paulo, Editora Sociología e Política, 1961; A. R., Nogueira, "O início da imigração nipônica para a lavoura cafeeira paulista", en Centro de Estudios Nipo-Brasileños, O Japonês em São Paulo e no Brasil, São Paulo, 1971, pp. 41-48. Orlando Sampaio Silva, "A presença de japoneses na zona Bragantina, Pará", Cadernos, Centro de Estudos Rudos Rurais e Urbanos (5), 1972, pp. 113-128; Francisca Isabel Schvrig Vieira, $O$ japonês na frente de expaução paulista, São Paulo, Editora de la Universidad de São Paulo y Livraria Pioneira, 1973; John T. Sasaki, "Japanese Inmigrant Farmers in Brazil", Migration News, 21 (1), enero-febrero de 1972, pp. 3-6. Así como también los trabajos de Jean Roche, A colonização alemã no Espirito Santo, Editora de la Universidad de São Paulo y Difusão Européia do Livro, São Paulo, 1968. Arpad, Szilvassy, "As possibilidades de desenvolvimento de uma região meridional; parte II, en Relações Humanas, 7 (20-21), agosto-diciembre de 1964, pp. 97-125; Elisabeth Vorcaro Horta, "Aspectos sociais do movimento imigratório da Itália para o Brasil", Revista de la Universidad de Minas Gerais (14), septiembre de 1964, pp. 84-106. 
a veces a privilegiar demasiado los rasgos culturales comunes más generales, o sea los rasgos de origen nacional más evidentes. Ese énfasis en los rasgos culturales de origen, muchas veces resulta también en que éstos son buscados de preferencia en las determinaciones locales, al igual que en los estudios orientados a la organización productiva de las áreas rurales de inmigración. En este caso se trata casi siempre de determinar la influencia de los inmigrantes en el desarrollo agrícola a través de los cultivos introducidos, de las técnicas agrícolas "traídas" de los lugares de origen, o también de hábitos, tradiciones culturales, familiares, religiosas, etcétera. El interés del conocimiento de estos aspectos no requiere enfatizarse. Pero la sobrestimación de su influencia puede resultar en la tentación de encontrar por todas partes la reproducción de la organización económica y de las técnicas productivas "traídas" por los inmigrantes. Así, se descuidan aspectos importantes para la explicación del desarrollo agrícola, como las condiciones locales de acceso a la posesión de la tierra, las relaciones de comercialización determinadas por las formas de inserción en la economía regional, el nivel previo de acumulación y las formas de creación del excedente, etc., en cuanto son destacados los hábitos y predisposiciones de los inmigrantes, explicados a partir de su origen.

El estudio de las migraciones internas es, en todo sentido, lo más interesante para la determinación de las relaciones de la dinámica poblacional y de la estructura agraria. En efecto, la mayor parte de los estudios realizados en el último decenio sobre las migraciones internas en Brasil, coinciden en apuntar la estructura agraria en las regiones de emigración como uno de los principales factores determinantes de los desplazamientos poblacionales.

En la medida en que el principal marco teórico de esos estudios parece ser el modelo de interacción entre los factores de repulsión en el origen y los de atracción en el destino de los flujos migratorios, las características económicas de las regiones rurales interesan en primer lugar desde el punto de vista de los obstáculos que representan para el crecimiento local y la fijación de la población." Sin embargo, al examinar más

- Como ejemplos de estudios sobre las migraciones internas que caracterizan los rasgos generales de las diferencias rurales - urbanas e interregionales, véanse Fausto Alves de Brito, "Migrações inter-regionais no Brasil", en Universidade Federal de Minas Gerais, Centro de Desenvolvimento e Planejamento Regional, Migraçoes internas e desenvolvimento regional, Belo Horizonte, CEDEPLAR, 1972, Vol. II, pp. 149-193; Pedro Pinchas Geiger, "Migrações internas e aspectos espaciais" en M. A. Costa (Comp), Migrações internas no Brasil, Rio de Janeiro, IPEA/INPEs, 1971, pp. 155-188; Pedro Pinchas Geiger, "Migraçōes inter-regionais e intra-regionais no Brasil", en Universidade Federal de Minas Gerais, Centro de Desenvolvimento e Planejamento Regional, Migrações internas y desenvolvimento regional, Belo Horizonte, CEDEPLAR, 1972, vol. II, pp. 27-71; Douglas H. Graham, "Padrōes de convergência e divergência do rescimento econômico regional e das migrações no Brasil, 1940-1960", Revista Brasileira de Economia, 23 (3), julio-septiembre de 1969, pp. 53-76; Douglas H. Graham y Sergio Buarque de Hollanda Filho, Migration, Regional and Urban Growth and Development in Brazil : A Selective Analysis of the 
de cerca la extensa lista de estudios sobre el tema se verifica un trata* miento más superficial de la economía agrícola de lo que sería deseable. A partir de la verificación empírica de la importancia cuantitativa de los flujos y del establecimiento de la relación entre diferencias de ingreso en los lugares de origen y destino, y, por otra parte, los procesos migratorios, la explicación se reduce casi siempre a hipótesis genéricas. Un conjunto de condiciones asociadas a la pobreza en las áreas de origen se considera suficiente para explicar la salida de contingentes poblacionales en busca de mejor remuneración, o mayores oportunidades en general. El primer reparo a esta explicación es que no pasa de una descripción, además de superficial. Si es un hecho establecido, se podría decir obvio, que las regiones rurales brasileñas son por lo general las más pobres del país, ${ }^{7}$ y que las migraciones de origen rural constituyen, desde hace muchos decenios, la principal corriente migratoria, es inevitable la asociación entre pobreza y migración. A tal punto que una puede ser tomada como indicador de la otra. Pero para que se pueda determinar cómo y por qué el campo se empobrece y de qué forma ese empobrecimiento puede significar una presión sobre el crecimiento poblacional, sería necesario ir más allá de la caracterización de la economía agrícola

Historical Record (1872-1970), Universidad de São Paulo, Instituto de Investigaciones Económicas, São Paulo, 1971; Douglas H. Graham y S. B. de Hollanda Filho, "Interregional and Urban Migration and Economic Growth in Brazil", en Universidade Federal de Minas Gerais, Centro de Desenvolvimento e Planejamento Regional, Migrações internas e desenvolvimento regional, Belo Horizonte, CEDEPLAR, 1972, vol. II, pp. 99-148; Gian S. Sahota, "An Economic Analysis of Internal Migration in Brazil", Journal of Political Economy, 76 (2, parte 1), marzo-abril de 1968, pp. 218-245. En relación a los factores de expulsión en las migraciones rurales-urbanas, véase también: Paulo Maciel, As migraçōes para o Recife; III: Aspectos econômicos, Recife, MEC-IJNPS, 1961. Algunos estudios sobre migraciones rurales-rurales analizan las motivaciones de los migrantes en las localidades de origen de la salida: Vania Porto Tavares, et al., "Fluxos de migraçōes internas", en Colonização dirigida no Brasil: suas possibilidades na região amazônica, Rio de Janeiro, IPEA/INPES, 1972, pp. 72-79; Thomas G. Sanders, Brazilian Interior Migration: There Frontier Cities on the Belém-Brasilia Highway, Hanover, NA, American Universities Field Staff, 1971, 10 pp. (Fieldstaff Reports, East Coast South America Series, 15 N: (2); Santa Helena, Bosco, y Antonio Jordão Netto, Migraçōes: Estudo especial sobre as migrações internas para o Estado de São Paulo $e$ seus efeitos, São Paulo (Estado), Secretaría de Agricultura, Departamento de Inmigración y Colonización, 1967; Antonio Jordão Netto, Aspectos econômicos e sociais das migrações internas para o Estado de São Paulo, São Paulo, 1973 (Mimeo).

7 Los indicadores de ingreso per capita, en ciertos casos, son usados de manera directa en la determinación del carácter rural o urbano de una región. Por ejemplo, en un modelo de simulación recién construido, se consideran rurales las regiones con ingresos inferiores a 200 dólares per capita y urbanas las demás. Véase Speridião Faissol, "Migraçōes inter-regionais no Brasil e suas relaçōes com o desenvolvimento nacional e regional : um modelo de simulação", en Universidade Federal de Minas Gerais, Centro de Desenvolvimento e Planejamento Regional, Migrações internas e desenvolvimento regional, Belo Horizonte, 'CEDEPLAR, 1972, vol. II, pp. 5-26. 
y de sus relaciones con la separación entre población y fuerza de trabajo. ${ }^{8}$ El análisis resuelve algunas cuestiones, en este caso, peró se está tan lejos de una formulación teórica definitiva como de una verificación empírica suficiente. En resumen, tales cuestiones podrían ser agrupadas en un conjunto de interrogantes que surgen de la lectura de estudios sobre migraciones internas en Brasil:

1. Cuáles son las características de la agricultura llamada de subsistencia $\mathrm{y}$ en qué medida y forma la pequeña producción actúa como elemento de fijación de poblaciones al margen del mercado de trabajo $o$, por el contrario, en la creación de excedentes poblacionales que tienden a ampliar el ejército industrial de reserva;

2. En qué medida y sentido el reciente desarrollo agrícola, con la expulsión de trabajadores residentes en las grandes propiedades, registrada en diferentes regiones del país, afectará la remuneración de la fuerza de trabajo. Si esa expulsión establece un nuevo tipo de mercado de trabajo agrícola, caracterizado por el contrato diario, a destajo, de los trabajadores que pasan a residir en suburbios de los centros regionales, no cabe duda que el valor de la fuerza de trabajo en general, podría afectarse por la modificación positiva de la productividad agrícola. Por otro lado, las nuevas fuentes de presión poblacional en las periferias urbanas ejercen con seguridad algún papel en la baja de la remuneración de los trabajadores;

3. Hasta qué grado las migraciones hacia las áreas de frontera constituyen una válvula de escape de las presiones poblacionales en las regiones de población más antigua, o en qué medida la recreación en esas áreas de una economía agrícola con reducidos insumos de capital, semejante al de las áreas de origen, puede significar un mero dislocamiento espacial del "atraso" y, por tanto, constituir una nueva fuente de presiones poblacionales;

4. En qué medida el crecimiento urbano de los centros medios, recién registrados, tiende a aliviar la presión poblacional sobre las grandes ciudades y facilita su desarrollo o, si por el contrario, pueden constituir un factor de desaceleración del propio desarrollo. Qué relación tendrían los mercados representados por los grandes centros y por las ciudades menores para los productos agrícolas con la dirección y grado de las transformaciones recientes en la agricultura.

${ }^{8}$ En estudio reciente se trata de establecer las cuestiones que afectan las interconecciones y el carácter social de los movimientos migratorios, a partir de un análisis de los cambios en la economía agrícola, en especial respecto a las relaciones entre producción de subsistencia y producción para el mercado. Sin embargo, se trata aún de la proposición de un punto de vista particular y de puntos por estudiarse: Juarez R. Brandão Lopes, "Desenvolvimento e migrações; uma abordajem histórico estrutural", Estudos CERRAP (6), octubre-diciembre de 1973, pp. 125-142. 
Este conjunto de cuestiones está ligado teóricamente a la dirección actual de la relación ciudad-campo: en qué medida la agricultura "atrasada" es un obstáculo para el desarrollo capitalista, sobre todo al determinar una limitación de mercado; o en qué medida ese atraso de la agricultura es producto del desarrollo capitalista y su constante renovación puede ser adecuada al actual proceso de concentración del capital. La salida para el atraso, representado por esos dilemas, sólo será posible en la medida que el estudio pueda dar cuenta del carácter contradictorio del reciente desarrollo agrícola, en el cual la "funcionalidad" del empobrecimiento de la población rural representa, al mismo tiempo, para la concentración del capital, un límite al proceso de concentración, en la medida en que determina la renovación permanente de una agricultura atrasada.

Los supuestos de esa discusión están presentes en una serie de estudios sobre desarrollo agrícola y estudios teóricos sobre el carácter de las relaciones de producción en la agricultura brasileña. Escaparía a los propósitos de la presente exposición comentar esos trabajos, una vez que la dinámica poblacional no es considerada en ellos de manera específica. Sin embargo, sería útil señalar que, a su vez, la lectura del debate sobre agricultura brasileña sugiere algunas indagaciones adicionales sobre el papel del crecimiento de la población y del consecuente aumento de la oferta de fuerza de trabajo en la determinación de la organización productiva en el campo. La cuestión central dentro de ésas es el carácter de los excedentes poblacionales, que hacen del campo el punto de partida de las corrientes migratorias. ¿Hasta qué punto esos excedentes están determinados por las condiciones de subsistencia en la agricultura o por los movimientos de concentración del capital? En otras palabras, se trata de saber en qué medida esos excedentes son producto del atraso o del desarrollo. 